\title{
Factores del contexto que afectan la competitividad de empresas lecheras del centro de Santa Fe
}

\author{
Acetta, P.M. ${ }^{1}$; Castignani, M.I. ${ }^{2}$ \& Rossler, N. ${ }^{2}$
}

\begin{abstract}
RESUMEN
El objetivo de este trabajo es realizar un análisis de los factores del contexto, macroentorno y entorno específico, que pueden influir en la competitividad de las empresas lecheras del centro de la provincia de Santa Fe. Para analizar el entorno general, se implementó el marco PESTEL y para analizar el entorno específico, se recurrió al modelo de las Cinco fuerzas de Porter. A partir de las oportunidades y amenazas detectadas, se proponen acciones que aportan a la definición de las distintas estrategias competitivas que podrían desarrollar las empresas a partir de sus recursos y capacidades. Un buen equilibrio entre los factores que generan riqueza, provocan empresas más atractivas y sustentables. El desafío que se presenta a las empresas lecheras es ser innovadoras y lograr resiliencia organizacional. Entre las oportunidades se destaca el influjo de las nuevas tecnologías de productos, procesos y servicios y las posibilidades de agregado de valor al producto y subproductos de los diferentes procesos. Entre las amenazas se destaca la fuerte competencia por recurso suelo, la figura del productor primario como tomador de precios de insumos y de la producción primaria, destacando la importancia de la escala y el agregado de valor a la producción.
\end{abstract}

Palabras clave: entorno general, entorno específico, ventajas competitivas, oportunidades, amenazas.

\footnotetext{
1.- Departamento de Ciencias Básicas. Facultad de Ciencias Agrarias (UNL). Kreder 2805. 3080HOF, Esperanza, provincia de Santa Fe, Argentina. Tel-fax +54 3496426400 (int. 263). Email: pacetta@fca.unl.edu.ar 2.- Departamento de Ciencias Sociales. Facultad de Ciencias Agrarias (UNL).

Manuscrito recibido el 2 de julio de 2021 y aceptado para su publicación el 20 de diciembre de 2021.
}

Acetta, P.M.; Castignani, M.I. \& Rossler, N. Factores del contexto que afectan la competitividad de empresas lecheras del centro de Santa Fe.

FAVE - Ciencias Agrarias 21 (1): 7-20 - CC BY-NC-SA 4.0 


\begin{abstract}
Context factors affecting the competitiveness of dairy companies in the center of Santa Fe.

The objective of this work is to carry out an analysis of the factors of the context, macroenvironment and specific environment, which may influence the competitiveness of dairy companies in the center of the province of Santa Fe. To analyze the general environment, the PESTEL framework was implemented and to analyze the specific environment, Porter's Five Forces model was used. Based on the opportunities and threats detected, actions are proposed that contribute to the definition of the different competitive strategies that companies could develop based on their resources and capabilities. A good balance between the factors that generate wealth, cause more attractive and sustainable companies. The challenge for dairy companies is to be innovative and achieve organizational resilience. Among the opportunities, the influence of new technologies for products, processes and services and the possibilities of adding value to the product and by-products of the different processes stand out. Among the threats, the strong competition for land resources stands out, as well as the figure of the primary producer as the price taker of inputs and primary production, highlighting the importance of scale and the addition of value to production.
\end{abstract}

Key words: general environment, specific environment, competitive advantages, opportunities, threats.

\section{INTRODUCCIÓN}

La lechería argentina tiene importantes desafíos. La falta de reacción frente a las condiciones cambiantes del mercado y sus riesgos, tendrán consecuencias negativas en términos competitivos a nivel sectorial, incluyendo fuertes variaciones de precios en el mercado doméstico, inversiones esporádicas, un portafolio estático de productos, un desarrollo oportunista de la capacidad comercial, limitadas oportunidades de crecimiento, aletargamiento del crecimiento doméstico, menor crecimiento de las exportaciones, llegando al riesgo de que en el largo plazo otros exportadores focalicen su llegada al mercado doméstico, a medida que desaparecen las barreras comerciales (Depetris de Guiguet et al., 2013). El COVID-19 ha acelerado tendencias preexis- tentes en los agronegocios. Cambios que se pensaba tomarían años se hacen presentes. El foco se encuentra ahora en la digitalización, medidas contra el cambio climático y localización o trazabilidad de la producción de alimentos (Foscarini, 2020).

Las provincias de Santa Fe, Córdoba, Buenos Aires y Entre Ríos, concentran el $84,97 \%$ de las explotaciones agropecuarias (EAPs) con actividad tambo. En esta zona, el sector lácteo está desarrollado y consolidado, con presencia de empresas procesadoras de productos lácteos, una variedad de servicios que atienden a todos los eslabones de la cadena y universidades e institutos de investigación vinculados a la actividad. Datos del Censo Nacional Agropecuario (CNA) 2002 (INDEC, 2002) indican que la cantidad de EAPs con actividad tambo a nivel nacional era de 15.250, de las 
cuales 4.020 pertenecían a la provincia de Santa Fe. Según el CNA 2018 de las EAPs relevadas, 5.995 declararon desarrollar actividad de tambo (INDEC, 2021a), de las cuales 1.727 corresponden a la provincia de Santa Fe, representando el 28,8\% del total del país.

En términos de producción, a lo largo del período 2008-2019, creció un 3,33\%. El crecimiento entre 1999 y 2019 es del 0,13\% (Bergero and Lugones, 2020) y, en comparación con el año 2020, el porcentaje mejora a 7,05\%. Esto es así porque el año 2020 cerró con una producción de 11.113 millones de litros de leche, con un incremento interanual del 7,44\% (OCLA, 2021). Según Consorcio Regional de Experimentación Agropecuaria (CREA), esta mejora estaría vinculada a un aumento en la producción individual de las vacas en ordeño. No obstante, el 2020 terminó un 7,9\% por debajo del año récord de producción, que fue el 2015 (OCLA, 2021).

En la provincia de Santa Fe, en 2020, se produjeron 2.412,5 millones de litros de leche (IPEC and Ministerio de Producción, Ciencia y Tecnología, 2021). Los conocimientos y la tecnología existentes, las condiciones agroecológicas y la consolidación y desarrollo del sector primario en la actualidad, permiten pensar que es posible lograr un aumento en los niveles de producción (más allá de los actuales).

El precio de la leche al productor para el mes de abril del 2021 fue de \$28,17 por litro según el Sistema Integrado de Gestión de la Lechería Argentina (SIGLeA). En tanto, el costo de producción fue de \$ 27,58, arrojando una tasa de rentabilidad al capital del 0,86\% (OCLA, 2021). La variación interanual abril 2021/abril 2020 del dólar mayorista BCRA fue de 41,2\%, índi- ce de precios al consumidor (IPC) fue de $46,3 \%$, el precio percibido por el productor según SIGLeA fue de 54,6\% y el costo de producción por litro de leche fue de 68,7\%. Esto muestra que, a pesar de que el precio se incrementa por encima del tipo de cambio y supera al índice inflacionario en la variación interanual, no logra compensar la suba del costo de producción que genera el incremento de los precios internacionales de los granos, fundamentalmente soja y maíz, que tienen una alta participación en los costos del tambo (afectando los rubros alimentación y alquileres) (OCLA, 2021). Considerando que las empresas del sector primario son precio aceptantes, queda expuesta la importancia que tiene para los empresarios del sector el seguimiento constante de su estructura de costos.

Para sobrevivir y tener éxito, una empresa debe desarrollar estrategias para contrarrestar de manera efectiva la rivalidad de los competidores dentro de su industria, la amenaza de nuevos entrantes y de los sustitutos, el poder de negociación de los clientes y de los proveedores, junto a su interacción explican por qué innovan y se mantienen competitivas las empresas ubicadas en determinadas regiones (Porter, 1996).

El objetivo de este trabajo es realizar un análisis de los factores del contexto, macroentorno y entorno específico, que orienten, a partir de sus recursos y capacidades, al desarrollo de estrategias competitivas para empresas lecheras del centro de la provincia de Santa Fe. 


\section{MATERIALES Y MÉTODOS}

El ambiente externo forma parte del desarrollo de una organización. Su análisis para la identificación de oportunidades y amenazas, junto al de las capacidades y recursos, permiten diagnosticar la posición competitiva de la empresa y formular estrategias que conlleven al logro de sus objetivos. La metodología propuesta por diversos autores del área de Dirección Estratégica de las empresas (Wheelen and Hunger, 2007; Thompson et al., 2013; Beristain and Guerras, 2016) plantea el estudio del contexto en dos niveles: entorno general, referido a los factores que afectan a la empresa desde un punto de vista global, y entorno específico, identificando las fuerzas competitivas derivadas del tipo de actividad que se desarrolla. Para analizar el entorno general, se implementó el marco PESTEL propuesto por Johnson y otros (2006). Se trata de un análisis estratégico para determinar el contexto actual en el que se mueven las organizaciones, se identifican los factores y se define el tipo de impacto (positivo o negativo) que poseen sobre las organizaciones: Políticos: de tipo gubernamental, que influyen en el desempeño de las empresas como la política fiscal, las restricciones al comercio y la industria, los aranceles; Económicos: tasas de cambio, tasas de interés, inflación, niveles de importación y exportación; Sociales: relacionados con las características básicas, preferencias, gustos y hábitos de consumo de la sociedad, renta total disponible, tasa de crecimiento de la sociedad y educación; Tecnológicos: tendencias que se producen en el área de sistemas informáticos y nuevas tecnologías, la nueva era digital; Ecológicos (o Ambientales): el cambio climático, las variaciones de las temperaturas y la conciencia ambiental; Legales: cambios en la normativa legal que afectan a las empresas. Para analizar el entorno específico se recurrió al modelo de las "Cinco fuerzas” de Porter (Porter, 1991), que permite analizar cinco factores determinantes de la competitividad: rivalidad entre competidores actuales, amenaza de competidores potenciales, poder de negociación de proveedores, poder de negociación de clientes y amenaza de los productos sustitutivos. Se enfoca en la determinación de los beneficios y riesgos que tiene un sector y ayuda a comprender sus fuentes de competencia, como mencionan Johnson y otros (2006), permitiendo desarrollar una estrategia de negocio, analizando la competencia mediante la comprensión de "fuerzas competitivas" que operan en un sector productivo y evaluando la presión de cada fuerza.

Se utilizaron fuentes de información secundaria (revisión y análisis de datos de censos y bibliografía acerca del tema de estudio). Además, se organizaron seis paneles de debate especializados donde hubo intercambio con profesionales vinculados al sector, directores de empresas del sector de los agronegocios, referentes de organizaciones de productores, director de observatorio rural, profesionales de institutos de tecnología. Estos paneles se centraron en temáticas como Dirección Estratégica de los Negocios Agroalimentarios, Gestión de las Personas en los Negocios Agroalimentarios, Bioeconomía, los Sistemas Agroalimentario. Cuatro de los paneles se realizaron de manera presencial y dos de manera virtual, con participación de alumnos de posgrado. Cada panel estuvo conformado por 10 a 15 personas. El método en cada panel fue el mismo: los especialistas presentaban los temas de interés y, luego, se abría el debate con los asistentes. 
A partir de la síntesis de los paneles y la información secundaria, los autores identificaron oportunidades y amenazas a las que se puedan enfrentar y, a partir de allí, se propusieron acciones para la definición de estrategias genéricas que podrían formular las empresas considerando su capacidad de respuesta (derivadas de sus fortalezas y debilidades) a los factores del entorno identificados.

La zona que abarca el estudio es la cuenca lechera central santafesina, que es una de las más importantes de Argentina y Latinoamérica, no solo por la cantidad de tambos y vacas en producción que posee, sino también por concentrar a las principales empresas elaboradoras y exportadoras de productos lácteos y por representar un dinamizador fundamental de las economías regionales (Gobierno de Santa Fe, 2021).

\section{RESULTADOS}

A continuación se presenta el análisis del entorno competitivo de la empresa, elaborado a partir de los análisis de PESTEL y "Análisis de las 5 fuerzas competitivas de Porter". Estos permiten determinar el atractivo de la industria a fin de visualizar la capacidad potencial de obtener beneficios e identificar las áreas que presentan oportunidades y amenazas, y se proponen la formulación de estrategias que permitan a las organizaciones mejorar su posición competitiva en la industria.

Análisis del entorno general: PESTEL

A través de la revisión de fuentes de información primaria y secundaria se identifican los factores y se define el tipo de impacto (positivo o negativo) que poseen sobre las organizaciones. En un paso posterior, cada empresa debería hacer su valoración en función de su capacidad de reacción.

En la Tabla 1 se resume el impacto positivo $\square$ o negativo $区$ de los factores analizados y posteriormente se describen.

Aspectos Políticos: Se destacan en este punto las políticas comerciales, como las restricciones cuantitativas o cuotas, el cierre de exportaciones, las retenciones (o tributos) a la exportación. La experiencia internacional deja a la Argentina como caso prácticamente único de una presión tributaria a sus ramas productoras de bienes exportables, lo cual le quita competitividad y dificulta el acceso a mercados (Calzada, 2020). Los programas de promoción se consideran como oportunidades para el sector. Ejemplos de estos son el PROBIOMASA (programa para la promoción de la energía derivada de la biomasa, para asegurar un suministro de energía limpia, confiable y competitiva) para abrir nuevas oportunidades agroforestales, estimular el desarrollo regional y contribuir a mitigar el cambio climático (FAO, MECON and MINAGRI, 2021) y el Programa RenovAr es un programa de abastecimiento de energía eléctrica a partir de fuentes renovables (FAO, 2019, 2020). Además, los programas de certificación de competencias laborales incentivan a los operarios a obtener un título oficial que garantice sus conocimientos del oficio en el que se desempeña; también al empleador, porque sabe que contrata a alguien capacitado (Ministerio de Trabajo, Empleo y Seguridad Social, 2017).

Aspectos Económicos: La devaluación de la moneda nacional, la incertidumbre respecto a los diferentes tipos de cambio, la elevada e imprevista inflación, está impactando directamente en la competitividad de las empresas, principalmente por la incerti- 
P. M. Acetta et al.

Tabla 1. PESTEL.

Table 1: PESTEL.

\begin{tabular}{|c|c|c|}
\hline POLÍTICO & ECONÓMICO & SOCIALES \\
\hline $\begin{array}{l}\text { 叉 Politicas comerciales } \\
\text { (restricciones cuantitativas, } \\
\text { cierre, retenciones a la } \\
\text { exportación) } \\
\text { 邓 Incertidumbre derivadas del } \\
\text { contexto político } \\
\square \text { Reintegros a la exportación } \\
\square \text { Programas de promoción }\end{array}$ & $\begin{array}{l}\text { D Devaluación de la moneda } \\
\text { nacional } \\
\text { 冈 Altas tasas de Inflación } \\
\text { Altas Tasas de interés } \\
\text { Tasas de crecimiento } \\
\text { económico negativas, PBI } \\
\text { Desempleo } \\
\text { Disminución del poder } \\
\text { adquisitivo } \\
\square \text { Demanda Global de } \\
\text { Alimentos en aumento }\end{array}$ & $\begin{array}{l}\square \text { C Consumidores: consumo } \\
\text { saludable, de alimentos } \\
\text { funcionales, superalimentos, } \\
\text { una mayor preferencia por lo } \\
\text { orgánico o ecológico } \\
\text { ■ Demanda creciente de } \\
\text { personal calificado asociado a } \\
\text { las nuevas tecnologías } \\
\square \text { Responsabilidad social } \\
\text { Empresarial }\end{array}$ \\
\hline TECNOLÓGICO & $\begin{array}{c}\text { ECOLÓGICO o } \\
\text { AMBIENTAL }\end{array}$ & LEGAL \\
\hline $\begin{array}{l}\text { Nuevas tecnologias y } \\
\text { digitalización: AgTech, IA, } \\
\text { blockchain, IOT, avances en } \\
\text { Robótica } \\
\text { 囚 Infraestructura regional } \\
\text { deficiente }\end{array}$ & $\begin{array}{l}\bigotimes \text { Adversidades climáticas } \\
\square \text { Disponibilidad de Recursos } \\
\square \text { Programas de buenas } \\
\text { prácticas agropecuarias } \\
\square \text { Agregado de valor a los } \\
\text { residuos pecuarios y biomasa } \\
\square \text { Legislaciones ambientales }\end{array}$ & $\begin{array}{l}\bigotimes \text { Presión impositiva alta } \\
\square \text { Legislación sobre la } \\
\text { competencia } \\
\square \text { Legislación Laboral }\end{array}$ \\
\hline
\end{tabular}

dumbre que genera al sector, que condiciona las inversiones, y por el impacto en los costos de producción. Las tasas de interés altas (OCLA, 2021) impactan en el costo de capital de la empresa y, en consecuencia, en su capacidad para obtener fondos e invertir en nuevos activos. El producto bruto interno (PBI) tuvo una caída de 9,9\% interanual en el acumulado de 2020 debido a la disminución de todos los componentes de la demanda. Se adjudica esta caída del producto a la pandemia por COVID 19, pero la escasez de recursos y algunos problemas macroeconómicos internos del país retrasan la recuperación (INDEC, 2021b).

El mercado de trabajo está debilitado, con una tasa de desempleo alta, en particular entre los jóvenes, sumado a una alta informalidad de la economía, lo que genera empleo precario para una gran parte de la población (Ernst and Mourelo, 2020). La pérdida de poder adquisitivo -en un año los salarios privados formales perdieron casi 5\% frente a la inflación (Bermúdez, 2021)determina el comportamiento del consumidor e impactan en un cambio en los hábitos de consumo, los clientes priorizan los productos en función del precio y su capacidad de compra.

La demanda global de alimentos impacta sobre las exportaciones, las proyecciones muestran que para alimentar la población mundial en 2050 sería necesario aumentar la producción de alimentos en un 70\% (FAO, 2009). La producción en los países en desarrollo tendría que duplicarse, implicando un aumento en la producción de varios productos básicos fundamentales. La reciente medida aprobada por China (Xinhua, 2021), sobre la relajación de sus medi- 
das de planificación familiar, permitiendo a sus ciudadanos tener un tercer hijo, aumenta las oportunidades para Argentina por el mayor consumo que genera.

Aspectos Sociales: En cuestiones productivas, la incorporación de las nuevas tecnologías requiere de mayor especialización y competencia por parte de los operarios. El factor humano se percibe cada vez más como un punto crítico y suele operar como una limitante a la incorporación de tecnologías (Castignani et al., 2012). Existe una tendencia de la demanda hacia el consumo saludable, de alimentos funcionales, superalimentos, una mayor preferencia por lo orgánico o ecológico. Por este motivo o por propia convicción, las empresas se desempeñan cada vez con mayor responsabilidad social, trabajando de forma sustentable en lo económico, lo social y lo ambiental, reconociendo los intereses de los distintos públicos con los que se relacionan, buscando la sustentabilidad a fin de no afectar (o beneficiar) a las generaciones futuras. Algunos indicadores permiten a los empresarios autoevaluarse en estos términos como el Indicador de Responsabilidad Social y Sustentabilidad para Empresas Agropecuarias (IARSE and BCCBA, 2020).

Aspectos Tecnológicos: Nuevas tecnologías y digitalización, buscando incrementos de eficiencia y mayor integridad de las cadenas de valor están hoy disponibles: Internet de las Cosas (IoT), tecnologías de sensores, manejo de información en la nube, edición de genes de plantas y semillas, inteligencia artificial (apoyada en los datos generados por la IoT, que posibilita el análisis predictivo y la identificación de patrones), el uso del blockchain (Foscarini, 2020). El concepto de AgTech abarca una amplia gama de nuevas tecnologías que son aplicadas a los sistemas agropecuarios per- mitiendo aumentar la productividad, la eficiencia y la rentabilidad (Aapresid, 2018).

Deficiencias en infraestructura básica, como caminos, desagües y electrificación (OCLA, 2021) y en conectividad y comunicaciones, redes de baja latencia, redes de alto ancho de banda, capacidad de procesamiento masivo, bases de datos, políticas nacionales en gestión de los datos. Hay pocas actividades que se pueden llevar a cabo de forma remota, debiéndose a la falta de conectividad de un número significativo de personas o a la dificultad de varios actores para acelerar su transformación digital (García Zaballos et al., 2020).

Aspectos Ecológicos o Ambientales: Fenómenos climáticos imprevistos, con probabilidades de ocurrencia cada vez más altas (sequías, inundaciones) y aparición de enfermedades actúan como una amenaza tanto para la sociedad como para las empresas (Siclari Bravo, 2020). La disponibilidad de recursos y condiciones ambientales son favorables para diversas producciones, incluso, son una ventaja competitiva respecto a otros actores del sector mundial. Lo que lleva a oportunidades de agregar valor a los residuos pecuarios y a la biomasa gracias a los avances en Bioeconomía (INTA, 2014). Los programas de buenas prácticas agropecuarias (BPAs) son una manera adecuada de producir y procesar los productos agropecuarios para una producción sana, segura y amigable con el ambiente (RED BPA, 2020).

Las legislaciones ambientales podrían causar un impacto en la evolución futura de las actividades productivas, dada la creciente tendencia hacia prácticas sostenibles, llevando a soluciones innovadoras que mejoren la competitividad del sector en el largo plazo (OECD and FAO, 2020) y que también pueden dejar fuera del sistema a aque- 
llos que no estén en condiciones de implementarlas. La Ley del Árbol, Ley 13.836 de la Provincia de Santa Fe, promueve la conservación del arbolado en todo el territorio provincial, generando un medio ambiente sano, equilibrado y apto para el desarrollo humano (Cámara de Senadores, 2018). La huella ambiental corporativa (HAC) es una medida multicriterio del comportamiento ambiental de una empresa (Garapen, Saila and Plangintza, 2017).

Aspectos Legales: Alta presión impositiva, como lo indica el índice FADA (Fundación Agropecuaria para el Desarrollo de Argentina) de junio 2021, el 61,8\% de lo que produce el campo es retenido por el Estado en términos de impuestos nacionales, provinciales y municipales. Además, los derechos de exportación impactan negativamente porque incrementan los recursos no coparticipables, reducen los coparticipables por reducción del impuesto a las ganancias y son recursos que salen de las zonas productivas (FADA, 2021).

La Ley 25.966 sobre la competencia, para productos de origen agrícola y alimentarios abarca las indicaciones geográficas y denominaciones de origen utilizadas para la comercialización de productos de origen agrícola y alimentarios, en estado natural, acondicionados o procesados (Senado y Cámara de Diputados, 2004). Una parte fundamental de toda empresa es un ambiente de trabajo seguro y confortable para crear condiciones de sanidad e higiene en todos los procesos productivos y de la persona. La Ley N²6.727 de Trabajo Rural, para trabajadores agrarios regula el salario, la jornada de trabajo, el transporte, la vivienda y los alimentos que deben recibir (Congreso de la Nación, 2011).

\section{Análisis del entorno específico: modelo} de las "Cinco fuerzas" de Porter

Se presentan un análisis de las cinco fuerzas de Porter aplicadas a empresas predominantemente lecheras del Centro de la Provincia de Santa Fe:

Nuevos Competidores: El know how del negocio, la incorporación de tecnologías de procesos y de información y el nivel de inversión en capital requerido, son barreras de entradas altas (oportunidad fuerte). La presión de esta amenaza es débil.

Por otro lado, existe competencia por el recurso suelo y por recursos alimenticios para los animales, que se ve afectada por la cantidad de actores en la cadena (ley de oferta/demanda). La aparición de nuevos pooles de siembra, como nuevos actores, que influyen en la negociación por los recursos, compitiendo por el factor tierra con la agricultura. En ambos casos se trata de presiones de amenazas fuertes.

Productos sustitutos: Por las nuevas tendencias en el consumo, el cuidado del medio ambiente, el bienestar animal, la alimentación vegana, están apareciendo productos sustitutos de lácteos que a fuerza de estrategias de marketing ganan espacio en las góndolas. El componente sustentable es un requisito cada vez más ponderado por los consumidores a la hora de elegir alimentos. El mercado relevante de producto en la primera fase de la cadena productiva láctea es la leche cruda, como materia prima elemental y fundamental para la industria de la leche y productos lácteos, sin posibilidades de sustitución en su demanda.

En los últimos años, la presencia de sustitutos lácteos de origen vegetal (bebidas a base de soja, almendra y otras) aumentó en muchas regiones del mundo. Las tasas de crecimiento de estos sustitutos son fuertes, a partir de una base baja, pero se tienen 
opiniones contradictorias sobre su impacto ambiental y sus beneficios relativos para la salud. Por tanto, prevalece la incertidumbre sobre cómo esto afectará la demanda de lácteos (OECD and FAO, 2020). La presión de esta amenaza es débil.

Poder de negociación de los proveedores: El productor se reconoce como pagador de los precios que los proveedores imponen, con un bajo poder de negociación. La escala le permite lograr, ocasionalmente, algunas bonificaciones por volumen en compra de semillas o agroquímicos. Además, hay muchos proveedores potenciales. La presión de esta oportunidad es fuerte.

Poder de negociación de los clientes: Las reglas de juego establecidas en el mercado lácteo, implican una gran asimetría estructural en el poder de negociación entre las partes. Generalmente el precio, las condiciones de entrega y los plazos de pago del producto resultan en favor de las usinas procesadoras de leche (Vértiz, 2018), lo que genera una reducción de la intensidad competitiva y en una cartelización en la fijación del precio de compra (Purciariello and Fusco, 2017). En el SIGLeA, la norma vigente sugiere (no obliga) que la leche se pague como mínimo en un $80 \%$ por atributos de calidad y no más de un $20 \%$ por bonificaciones comerciales. Las bonificaciones comerciales en promedio llegan al 18\%, indicando que un alto porcentaje del valor de la leche cruda surge de su calidad (OCLA, 2021). El volumen de leche entregado por la empresa a la industria podría generar alguna situación de poder de negociación para el empresario productor de leche o para la empresa procesadora de la materia prima. En general, a mayor cantidad de leche mayor es el poder de negociación del precio del empresario productor de leche. La presión de esta oportunidad es débil.
Rivalidad y competencia de mercado: La rivalidad entre competidores está en el centro de las fuerzas y es el elemento más determinante del modelo de Porter, porque define la rentabilidad de un sector: cuanto menos nivel de competencia se verifique en un sector, normalmente será más rentable y viceversa. El sector de producción y el industrial conforman un mercado de tipo oligopsonio (Cisneros, 2002), el cual es una situación de competencia imperfecta porque ocurre cuando existen pocos compradores para un mismo producto. El precio al productor primario es sensible a lo que ocurre con la oferta y demanda de materia prima. La oferta está conformada por la producción de leche cruda del sector primario y la demanda por los consumidores locales y las ventas externas. El sector de transformación de leche cruda tiene una oferta de productos fija o prácticamente fija (se compra toda la materia prima producida dado que no es fácilmente exportable en su estado crudo) mientras que enfrenta una demanda variable. Por ello, las variaciones de demanda de productos lácteos se ajustan vía precios (Lema and Gallacher, 2004). Amenaza fuerte.

Innovaciones en tecnología permiten mayores índices de eficiencia de producción a nivel tambo, así como también a aumentar la calidad de la leche destinada a sus distintos usos, lo que permite mejorar los precios obtenidos por la leche cruda (Lema and Gallacher, 2004) y, de esta manera, una posición superior con respecto a los rivales. La oportunidad de esta presión es fuerte.

La relación de la lechería con la agricultura, con precios agrícolas favorables, incentivan a los productores a dedicar mayores recursos a la producción de granos (Castignani, 2003). El corrimiento de 
las fronteras agrícolas desplazó a algunos productores lecheros hacia zonas menos productivas y expulsando a otros del sector (Larrea et al., 2020). La amenaza es fuerte.

\section{Algunas acciones para definir estrate- gias}

Finalmente, de las oportunidades y amenazas identificadas a partir de los análisis del entorno general y específico del sector se proponen acciones para la definición de estrategias genéricas que podrían formular las empresas a partir de sus fortalezas y debilidades. La estrategia genérica competitiva sirve como marco del resto de las estrategias de la empresa, apoyándose en un conjunto adecuado de recursos, conocimientos y capacidades competitivas (Thompson et al., 2013). Michael Porter (Porter, 1985), en su análisis sobre las estrategias genéricas de las empresas diferencia dos tipos de ventaja competitiva, cada una ubica a la empresa en lugares distintos de su mercado y ambiente competitivo. Algunas acciones que podrían llevarse a cabo para cada estrategia son:

Estrategias de liderazgo en costos: $\mathrm{El}$ know how del negocio, la adopción de las nuevas tecnologías disponibles en el mercado (robotización, genética) y la implementación de procesos que lleven a una lechería de precisión (estrategias pastoriles, planificación de los recursos), permitirá a la empresa aumentar su eficiencia y reducir costos.

La participación de grupos o asociaciones de productores para la compra de insumos o contratación de servicios se plantea como estrategia comercial para la reducción de costos ya que permitiría mejorar el poder de negociación, lograr mejores precios por volumen o tener mejor acceso al mercado.
Estrategias de diferenciación de producto: Diferenciar el producto ofrecido, a través del agregado de valor de residuos (efluentes, por ejemplo), disminuyendo la medición de huella de carbono y promoviendo la responsabilidad social, con sellos de normas ambientales.

Enriquecer leche a través de un proceso natural como la dieta de los animales, obteniéndose leche y productos derivados con propiedades antioxidantes y preservada de los efectos de la oxidación, protegiendo estos productos a través de denominaciones de origen o marcas.

Estrategias de segmentación de mercado: Las nuevas oportunidades en avances tecnológico, demanda de productos diferenciados en calidad social y ambiental en un segmento determinado del mercado, exige un producto diferenciado según a los requerimientos específicos de cada segmento objetivo. Un ejemplo podría ser el de la leche orgánica para la industria alimenticia, resaltando además oportunidades en el área de los bionegocios, agregando valor al producto y sus subproductos.

Argentina ha desarrollado una alta competitividad por haber incorporado ciencia, tecnología y diseños organizacionales y de gestión empresarial que le permiten competir con los mejores a nivel global (Vilella, 2020). La bioeconomía es una perspectiva útil para identificar opciones y guiar las decisiones sobre los futuros senderos de desarrollo (Trigo, 2015). Además, es necesario considerar nuevas formas de generación y aplicación de conocimientos y un nuevo marco institucional en materia de políticas públicas, productivas, tecnológicas, regulatorias, sociales, educativas, comerciales y de infraestructura, entre otros (Lengyel and Zanazzi, 2020). 


\section{CONCLUSIONES}

En un contexto como el actual, en el cual muchas de las variables que forman parte de la función de beneficios del productor exhiben una inestabilidad creciente (precios internacionales y variables climáticas, por ejemplo), amenazando la continuidad en la actividad de muchos productores, surge la importancia del término "competitividad”. Una empresa competitiva tiene la habilidad para capturar las oportunidades que se le presentan brindando la posibilidad de mejorar y hasta reorientar su negocio. La bioeconomía constituye una gran oportunidad en este sentido.

El análisis del entorno general y específico, permitieron identificar oportunidades y amenazas de empresas predominantemente lecheras del Centro de Santa Fe y sus factores del contexto que determinan su competitividad, para considerarlas en futuros planes estratégicos y acciones que propicien el agregado de valor del sector y su continuidad. Queda expuesta la importancia de formular e implementar estrategias adecuadas y en permanente innovación. La competitividad de las empresas se centra en las ventajas competitivas que desarrolle internamente y en los condicionamientos externos. Para construirla y mantenerla se debe combinar el análisis de la industria con el desarrollo y evaluación de los recursos internos de las empresas. La determinación de los factores que inciden en la competitividad de una empresa es un aspecto fundamental para su proceso de medición y determinación de la estrategia competitiva.

La producción de leche del país, y del centro de Santa Fe en particular, junto a su industrialización ofrecen posibilidades para incrementar la medida de circularidad de los sistemas productivos. Pensando en la construcción de un futuro sustentable desde el punto de vista productivo y social, es necesario considerar el planteo de estrategias mejoradoras, que contemplen el cubrimiento de las necesidades detectadas respecto de recursos de capital de producción, instalaciones, equipos y maquinarias, para cada organización en particular. El rol del Estado y de las organizaciones del medio, como asociaciones de productores, cooperativas, entidades financieras, serán claves en términos de la asistencia para facilitar el acceso a capital. Un buen equilibrio entre los factores que generan riqueza, provocan empresas más atractivas y sustentables. El desafío que se presenta a las empresas agropecuarias y, en particular las empresas lecheras, pasa por ser innovadoras y el desarrollo de resiliencia organizacional.

\section{BIBLIOGRAFÍA}

Aapresid. 2018. Sobre Agtech. Available at: https://agtech.org.ar/ (Accessed: 10 June 2021).

Bergero, P. and Lugones. 2020. Análisis de la evolución del sector lácteo en Argentina durante el período 2008-2019. Rosario: BCR. Available at: http://www.bcr.com.ar (Accessed: 10 June 2021).

Beristain, J. and Guerras, L. 2016. La dirección estratégica de la empresa. 2nd edn. España: Thomson Reuters Civitas.

Bermúdez, I. 2021. Suba de precios. Available at: https://www.clarin.com/ (Accessed: 10 June 2021). 
Calzada, J. 2020. Algunas consideraciones respecto de cómo los Derechos de Exportación impactan en el interior y en las economías regionales. Informativo semanal 1946. Rosario: BCR. Available at: https://www.bcr. com.ar/es/print/pdf/node/78072 (Accessed: 10 June 2021).

Cámara de Senadores. 2018. 'Ley Provincial 13836/2018'. Gobierno de Santa Fe. Available at: https://www.santafe.gov.ar/index.php/ web/content/download/252277/1326746/file/ Ley\%2013836.pdf (Accessed: 10 June 2021).

Castignani, M.I. 2003. Análisis de la oferta de productos y demanda de insumos de empresas lecheras argentinas a partir de la función de beneficios. Magister. Universidad Católica de Chile. Available at: https://www.fca. unl.edu.ar/tictambo/web/docs/TesisCastignani.pdf (Accessed: 2 May 2021).

Castignani, M.I. et al. 2012. 'Los recursos humanos en la empresa lechera’, VII Jornadas Interdisciplinarias de Estudios Agrarios y Agroindustriales, p. 19. Available at: https:// www.fca.unl.edu.ar/tictambo/web/docs/RecHumanosEmpresaLechera.pdf (Accessed: 25 March 2021).

Cisneros, R.D. 2002. El sector lácteo argentino. Grado. UNMDP.

Congreso de la Nación. 2011. Ley 26.727. Trabajo Agrario. Available at: https://www.argentina.gob.ar/ (Accessed: 12 May 2021).

Depetris de Guiguet, E. et al. (2013) Cambios y Tendencias en las Políticas Lecheras de los Principales Participantes del Mercado Internacional. UNL. IECAL UNL.

Ernst, C. and Mourelo, E.L. 2020. La COVID-19 y el mundo del trabajo en Argentina. Informe técnico. OIT, p. 20. Available at: https://www.ilo.org/wcmsp5/groups/public/---americas/---ro-lima/---ilo-buenos_aires/ documents/publication/wcms_740742.pdf (Accessed: 20 April 2021).
FADA. 2021. Índice FADA de Junio 2021. Available at: https://fundacionfada.org/ (Accessed: 20 June 2021).

FAO. 2009. 'La agricultura mundial en la perspectiva del año 2050', Foro de eventos de alto nivel. Como alimentar al mundo 2050, p. 4. Available at: https://www.fao.org/fileadmin/templates/wsfs/docs/Issues_papers/ Issues_papers_SP/La_agricultura_mundial. pdf (Accessed: 1 April 2021).

FAO. 2019. Estudio de cuencas de biogás. 4. Available at: http://www.fao.org.

FAO. 2020. Modelo de negocio de aprovechamiento energético de biogás en tambos. Available at: http://www.probiomasa.gob. ar/_pdf/13-Modelo-negocio-biogas-tambos. pdf (Accessed: 22 May 2021).

FAO, MECON and MINAGRI. 2021. PROBIOMASA. Available at: http://www.probiomasa.gob.ar/ (Accessed: 17 May 2021).

Foscarini, G. 2020. Post-pandemia, el mundo de los agronegocios afronta una aceleración de tendencias, BID. Available at: https://idbinvest.org/.

Garapen, E., Saila, E.A. and Plangintza, L. 2017. 'Guía Metodológica para la aplicación de la Huella Ambiental Corporativa’, p. 52. Available at: https://www.euskadi.eus/contenidos/documentacion/huella_ambiental/es_ def/adjuntos/Guia_huella_ambiental_CASTdef.pdf (Accessed: 5 December 2020).

García Zaballos, A. et al. 2020. 'El impacto de la infraestructura digital en las consecuencias de la COVID-19 y en la mitigación de efectos futuros'. BID. Available at: https:// idbinvest.org/.

Gobierno de Santa Fe. 2021. Lechería y política láctea. Available at: https://www.santafe.gob. ar/ (Accessed: 17 June 2021). 
IARSE and BCCBA. 2020. Indicadores de Responsabilidad Social y Sustentabilidad para Empresas Agropecuarias. Available at: https://indicagro.bccba.org.ar/ (Accessed: 21 March 2021).

INDEC. 2002. Censo Nacional Agropecuario 2002. Available at: https://sitioanterior.indec. gob.ar/cna_index.asp (Accessed: 5 October 2020).

INDEC. 2021a. Censo Nacional Agropecuario 2018. Resultados definitivos. INDEC. Buenos Aires. Available at: https://www.indec. gob.ar/ftp/cuadros/economia/cna2018_resultados_definitivos.pdf (Accessed: 10 May 2021).

INDEC. 2021b. 'Informe de avance del nivel de actividad. Cuarto trimestre de 2020’, 5(51), p. 21. Available at: https://www.indec.gob. ar/uploads/informesdeprensa/pib_03_21B1D23916BF.pdf (Accessed: 10 May 2021).

INTA. 2014. 'Energías renovables'. Available at: https://inta.gob.ar/.

IPEC and Ministerio de Producción, Ciencia y Tecnología. 2021. Sector Lácteo Provincia de Santa Fe. Santa Fe: IPEC. Available at: http:// www.estadisticasantafe.gob.ar/wp-content/ uploads/sites/24/2021/05/SectorL\%C3\%A1cteoSF-0321.pdf (Accessed: 1 June 2021).

Johnson, G., Scholes, K. and Whittington, R. (2006) Dirección Estratégica. 7th edn. Madrid: Pearson Educación.

Larrea, Á.T. et al. 2020. 'Caracterización de las explotaciones lecheras de La Pampa’, 5, p. 12. Available at: http://www.ayv.unrc.edu.ar/ ojs/index.php/Ab_Intus/article/view/132/89 (Accessed: 11 November 2020).

Lema, D. and Gallacher, M. 2004. 'Análisis de la industria láctea: competitividad y relaciones'. Trabajo realizado para CIL (Centro de la Industria Lechera).
Lengyel, M. and Zanazzi, L. 2020. Bioeconomía y desarrollo en la Argentina. DOSIER CIECTI 4. Ciudad Autónoma de Buenos Aires: CIECTI, p. 64. Available at: http://www. ciecti.org.ar/wp-content/uploads/2020/10/ Dosier-4_V06.pdf (Accessed: 17 May 2021).

Ministerio de Trabajo, Empleo y Seguridad Social. 2017. Certificación de competencias laborales. Available at: https://www.argentina. gob.ar/trabajo/formacionycapacitacion/certificacioncompetencias (Accessed: 10 June 2021).

OCLA. 2021. Observatorio de la cadena Láctea Argentina. Available at: https://www.ocla. org.ar/ (Accessed: 20 June 2021).

OECD and FAO. 2020. OCDE-FAO Perspectivas Agrícolas 2020-2029. PAris: OECD, p. 352. Available at: https://www.oecd-ilibrary. org/agriculture-and-food/ocde-fao-perspectivas-agricolas-2020-2029_a0848ac0-es;jsessionid=RwJjthYBJWZ_cytxBckDqu1q.ip10-240-5-49 (Accessed: 19 May 2021).

Porter, M. 1991. Estrategia Competitiva. Argentina: Rei Argentina SA.

Porter, M.E. 1985. Competitive advantage: creating and sustaining superior performance. New York : London: Free Press ; Collier Macmillan.

Porter, M.E. 1996. 'What is strategy?', Harvard Business Review, 6.

Purciariello, A. and Fusco, M. 2017. 'RIESGO DE PRECIO: ANÁLISIS DE LOS FUNDAMENTALS DEL PRECIO DE LA LECHE ABONADO AL PRODUCTOR EN ARGENTINA', 1, p. 20. Available at: http://www.economicas.uba.ar/wp-content/uploads/2016/02/Purciariello-A.-Fusco-M.-Analisis-de-los-fundamentals-del-precio-de-la-leche-abonado-al-productor-en-Argentina.pdf (Accessed: 26 April 2021). 
RED BPA. 2020. Red de Buenas Prácticas Agropecuarias. Available at: https://redbpa.org.ar/ (Accessed: 10 March 2021).

Senado y Cámara de Diputados. 2004. Ley 25.966. Available at: http://servicios.infoleg. gob.ar/ (Accessed: 25 March 2021).

Siclari Bravo, P.G. 2020. 'Amenazas de cambio climático, métricas de mitigación y adaptación en ciudades de América Latina y el Caribe', Documentos de Proyectos(LC/ TS.2020/185), p. 89.

Thompson, A.A. et al. 2013. Administración estratégica. México: McGraw-Hill.

Trigo, E. 2015. La bioeconomía como modelo de desarrollo: oportunidades y desafíos. Available at: http://www.maizar.org.ar/vertext.php?id=475 (Accessed: 2 August 2020).
Vértiz, P. 2018. El complejo lácteo argentino: integración subordinada de la producción primaria a la dinámica del capital agroindustrial (período 2002-2015). Doctor en Ciencias Sociales. Universidad Nacional de La Plata. doi:10.35537/10915/68855.

Vilella, F. 2020. Por qué la bioeconomía argentina ha desarrollado alta competitividad a nivel global. Available at: https://www.clarin.com/ rural/bioeconomia-argentina-desarrollado-alta-competitividad-nivel-global_0_EJ_ udxzrn.html (Accessed: 14 September 2020).

Wheelen, T.L. and Hunger, D. 2007. Administración Estratégica y Política de Negocios. 10th edn. México: Pearson Ed.

Xinhua. 2021. China releases decision on threechild policy, supporting measures. Available at: http://www.xinhuanet.com/english/2021-07/20/c_1310072750.htm (Accessed: 4 December 2021). 